\title{
Liverworts of Alagoas State, Brazil
}

\author{
Lisi Dámaris Pereira Alvarenga ${ }^{1,2}$, Juliana Rosa do Pará Marques de Oliveira ${ }^{1}$, Mércia Patrícia Pereira Silva ${ }^{1}$, \\ Sarah Oliveira da Costa ${ }^{1}$ and Kátia Cavalcanti Pôrto ${ }^{1}$
}

Received: August 17, 2007. Accepted: November 29, 2007

\begin{abstract}
RESUMO - (Hepáticas do Estado de Alagoas, Brasil). No presente trabalho, são compiladas as espécies de hepáticas ocorrentes no Estado de Alagoas, resultantes de informações disponíveis em catálogos e trabalhos anteriores e do inventário inédito de uma importante unidade de conservação do Nordeste, a Estação Ecológica (EsEc) Murici (9¹1'05” - 9¹6 48”'S; 3545'20” - 3555'12”'O). São referidas para Alagoas 116 espécies de hepáticas, tendo 106 delas ocorrido na EsEc Murici. Setenta e oito espécies são genuinamente novas ocorrências para o Estado de Alagoas, sete das quais são novas também para a região Nordeste do Brasil. São fornecidos dados sobre a distribuição geográfica das espécies no Brasil e no mundo, e adicionalmente, comentários taxonômicos e ecológicos das espécies que são novas ocorrências para o Nordeste.
\end{abstract}

Palavras-chave: Hepáticas, Floresta Atlântica, Nordeste do Brasil, brioflora

\begin{abstract}
Liverworts of Alagoas State, Brazil). A list of liverworts from Alagoas State was compiled and is presented here. The list is based on catalogues and previous papers as well as the results of an unpublished survey carried out at the Murici Ecological Station (EsEc), an important protected area of the Brazilian Northeast ( $9^{\circ} 11^{\prime} 05^{\prime \prime}$ - 9 $\left.9^{\circ} 16^{\prime} 48^{\prime \prime} \mathrm{S} ; 35^{\circ} 45^{\prime} 20^{\prime \prime}-35^{\circ} 55^{\prime} 12^{\prime \prime W}\right)$. One-hundred and sixteen liverworts have been recorded for the state of Alagoas, of which 106 occur at EsEc Murici. Seventy-eight are new occurrences for Alagoas, and seven of these are also new occurrences for northeastern Brazil. Data on geographic distribution in Brazil and worldwide is given here, in addition to ecological and taxonomic comments on the species that are new occurrences for the Northeast region.
\end{abstract}

Key words: liverworts, Atlantic Forest, northeastern Brazil, bryophyte flora

\section{Introduction}

The bryophyte flora of Alagoas is one of the most poorly studied of the Brazilian Northeast. To date, no systematic survey has been performed and all references come from sparse and sporadic collections whose results have been put together in Yano's catalogues (1981; 1984; 1989; 1995; 2006).

Recently, Pôrto et al. (2006) carried out an inventory in four Atlantic Rain Forest remnants, recording 76 species of bryophytes - liverworts (31 spp.) and mosses (45 spp.) - while Yano \& Peralta (2007) increased this number by publishing 36 new occurrences (26 mosses and 10 liverworts) for Alagoas. Yet species richness in Alagoas is still low when compared to other states like Pernambuco and Bahia, which house more than 300 spp. (Pôrto \& Germano 2002; Bôas-Bastos, S.B.V., personal communication).

Additionally, Alagoas' bryophyte flora is now increased by the survey carried out at the Murici Ecological Station (EsEc), one of the main Atlantic Forest remnants of northeastern Brazil. EsEc Murici was created in 2001 with $6,116.43$ ha and is considered a priority area for biodiversity conservation in Brazil and worldwide (Brasil - MMA 2000). The survey resulted in a list of 181 species, of which 106 are liverworts, 72 are mosses, and three await special taxonomic treatment. This paper focuses only on liverworts, while mosses will receive attention in another publication.

This paper presents a compilation of liverwort species occurring in Alagoas State, bringing together published information (catalogues) and results from the EsEc Murici survey. Moreover, it provides data about the species' geographic distribution in Brazil and in the world, as well as taxonomic and ecological comments on the new occurrences for the northeastern region.

\section{Material and methods}

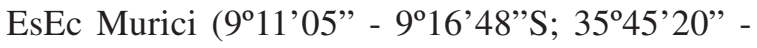
$\left.35^{\circ} 55^{\prime} 12^{\prime \prime} \mathrm{W}\right)$ is part of Murici and Messias

\footnotetext{
${ }^{1}$ Universidade Federal de Pernambuco, Departamento de Botânica, Laboratório Biologia de Briófitas, CCB, Av. Prof. Moraes Rêgo s.n., Cidade Universitária, 50670-901 Recife, PE, Brasil

2 Corresponding Author: lisidamaris@yahoo.com.br
} 
municipalities, $50 \mathrm{~km}$ from Maceió, capital of the state of Alagoas (Moura 2006). The elevation of the reserve ranges between $200-650 \mathrm{~m}$, and the area has warm, humid wheather, with a dry summer and a wet autumnwinter season. The mean annual precipitation and temperature are 2,167.7 and $24^{\circ} \mathrm{C}$, respectively (National Institute of Meteorology, data for the period from 1961 to 1990). The vegetation cover is classified as Submontane Open Humid Atlantic Rain Forest (Veloso et al. 1991).

The botanical material studied consists of epiphyllic (leaf inhabiting), epiphytic (bark inhabiting) and epixyllic (decaying wood inhabiting) samples collected from the understory and canopy. The material was examined in the laboratory; identification and taxonomic and ecological commentaries were based on the following literature: Fulford (1963; 1966; 1968; 1976), Stotler (1970), Kron (1988), Reiner-Drewald \& Goda (2000), Gradstein \& Costa (2003), and Dauphin (2003). Occasionally the identification of specimens was confirmed by specialists. The following literature was consulted to compile the information on geographic distribution: Yano (1984; 1989; 1995; 2004; 2005; 2006), Bastos \& Yano (2004; 2006), Câmara \& Vital (2004), Câmara \& Costa (2006), Costa \& Silva (2003), Costa et al. (2005), Ganacevich \& Mello (2006), Germano \& Pôrto (2005), Gradstein \& Costa (2003), Joyce et al. (2006), Molinaro \& Costa (2001), Oliveira et al. (2006), Paixão \& Mello (2006), Patrus \& Starling (2006), Pôrto et al. (2006), Valente \& Pôrto (2006a; 2006b), Vilas Bôas-Bastos, Bastos \& Ballejos (2006), Yano \& Bordin (2006), Yano \& Câmara (2004), Yano \& Costa (2000), and Yano \& Peralta (2005; 2006; 2007). The taxonomic classification adopted was that presented by Crandall-Stotler \& Stotler (2000). All samples studied are registered and deposited in the UFPGeraldo Mariz Herbarium, at the Federal University of Pernambuco.

The Alagoas liverwort species listed that did not occur in EsEc Murici where found in Yano's catalogues (1981; 1984; 1989; 1995; 2006), Gradstein \& Costa (2003), and Pôrto et al. (2006).

\section{Results and discussion}

One-hundred sixteen species of liverworts were recorded for Alagoas. Seventy-eight species are new occurrences for Alagoas state, occurring exclusively at EsEc Murici, and seven species are new occurrences for the Brazilian Northeast as well. The other 38 species had been previously cited in catalogues and other papers. The taxa are listed in alphabetical order by family and species and include locality, substrata and forest strata (understory or canopy) in the case of EsEc Murici, and geographic distribution in Brazil and in the world (Tab. 1).

Most of the species had typically Neotropical (59\%), Pantropical (16\%) and African-American (7\%) geographic distributions. Lejeuneaceae is by far the richest family in the state, with 83 species. This obviously is because the flora presented here comes mainly from Atlantic Rain Forest. It is known that this family used to be predominant in the flora of tropical regions, especially in forest habitats (Gradstein et al. 2001).

Geographic distribution details, taxonomic and ecological comments and ilustrations of the main characters of the new occurrences for Northeast Brazil are presented below.

\section{ANEURACEAE}

Aneura pinguis (L.) Dumort., Comment. Bot. 115. 1822.

Fig. 1-2

Material examined: BRAZIL. Alagoas: Murici, 2/XII/2004, Pôrto (UFP 45851); ib. 2/XII/2004, Pôrto (UFP 45850).

Description: Schuster (1992).

Comments: this species was placed within Riccardia S.F. Gray genus until recent decades. However, it can be distinguished from this genus because of at least two clear characters on gametophytes: 1 ) thallus less ramified, darker and wider, reaching up to $8 \mathrm{~mm}$ while Riccardia spp. reaches no more than $3 \mathrm{~mm}$ wide and; 2) many minutely dispersed oil bodies inside the cells. Aneura pinguis is easily recognized in the field even when sterile, due to its conspicuous and lustrous thallus, green to yellowish-green in color. Of the Aneura Dumort. species occurring in Brazil, A. pinguis is the only one with 10-18 (20) cell thickness in the middle (transversal section); the others are thinner, up to 9 cells thick. In Brazil, this species was only known for the North, Central and Southeast regions. It is sub-cosmopolitan and occurs at altitudes that vary from sea level to $800 \mathrm{~m}$. It was found growing on decaying wood in EsEc Murici.

\section{LEJEUNEACEAE}

Aphanolejeunea asperrima Steph., Sp. Hepat. 5: 859. 1916.

Fig. 3-6

Material examined: BRAZIL. Alagoas: Murici, 2/XII/2004, Pôrto (UFP 45822); ib. 2/XII/2004, Pôrto (UFP 45823); ib. 2/XII/2004, Pôrto (UFP 45825); ib. 2/XII/2004, Pôrto (UFP 45829); ib. 2/XII/2004, Pôrto (UFP 45830); ib. 2/XII/2004, Pôrto (UFP 45833); ib. 2/XII/2004, Pôrto (UFP 45845); ib. 2/XII/2004, Pôrto (UFP 45831); ib. 2/XII/2004, Pôrto (UFP 45842); ib. 2/XII/2004, Pôrto (UFP 45844). 


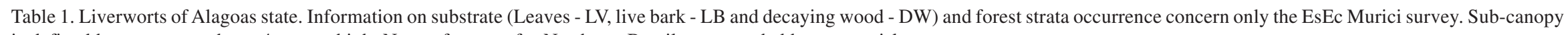
is defined here as strata above 4 meters high. New references for Northeast Brazil are preceded by an asterisk.

\begin{tabular}{|c|c|c|c|c|c|}
\hline Taxa & Locality & Substrata & Forest strata & World distribution & Distribution in Brazil \\
\hline \multicolumn{6}{|l|}{ MARCHANTIOPHYTA } \\
\hline \multicolumn{6}{|l|}{ ANEURACEAE } \\
\hline *Aneura pinguis (L.) Dumort. & EsEc Murici & DW & understory & Subcomsmopolitan & AM, MG, MS, RJ, SP \\
\hline Riccardia amazonica (Spruce) S.W. Arnell & EsEc Murici & DW & understory & Africa and America & AC, AM, AP, ES, MS, PA, PE, RJ, RS, SP \\
\hline \multicolumn{6}{|l|}{ BRYOPTERIDACEAE } \\
\hline Bryopteris diffusa (Sw.) Nees & $\begin{array}{l}\text { EsEc Murici, Messias, } \\
\text { Inião dos Palmares, and } \\
\text { Boca da Mata }\end{array}$ & LB & understory & Neotropical & $\begin{array}{l}\text { AL, AM, BA, CE, ES, MG, MT, PA, PE, PR, } \\
\text { RJ, RS, SC, SE, SP }\end{array}$ \\
\hline Bryopteris filicina (Sw.) Nees & EsEc Murici & LB, DW, LV & $\begin{array}{l}\text { understory, } \\
\text { sub-canopy, canopy }\end{array}$ & Neotropical & $\begin{array}{l}\text { AL, AM, BA, CE, ES, GO, MG, MS, MT, PA, } \\
\text { PE, PR, RJ, RR, RS, SC, SP }\end{array}$ \\
\hline \multicolumn{6}{|l|}{ CEPHALOZIELLACEAE } \\
\hline $\begin{array}{l}\text { Cylindrocolea rhizantha (Mont.) R.M. Schust. } \\
\text { CORSINIACEAE }\end{array}$ & EsEc Murici & DW & understory & Neotropical & AC, BA, ES, PE, RJ, SP \\
\hline Cronisia paradoxa Berk. & Junqueiro & - & - & Neotropical & AL, BA, CE, ES, GO, MT, SP \\
\hline \multicolumn{6}{|l|}{ GEOCALYCACEAE } \\
\hline Lophocolea liebmanniana Gottsche & EsEc Murici & LB, DW & understory & Neotropical & AC, AM, PA, PE, MT, SP \\
\hline L. martiana Nees & EsEc Murici & DW & understory & Africa and America & $\begin{array}{l}\text { AM, AP, BA, CE, ES, MG, PA, PE, PR, RJ, RS, } \\
\text { SC, SE, SP }\end{array}$ \\
\hline \multicolumn{6}{|l|}{ JUBULACEAE } \\
\hline Frullania apiculata (Reinw. et al.) Nees & EsEc Murici & $\mathrm{LB}, \mathrm{DW}$ & $\begin{array}{l}\text { understory, sub-canopy, } \\
\text { canopy }\end{array}$ & Pantropical & AM, BA, GO, PA, PE, RJ, SP \\
\hline F. brasiliensis Raddi & EsEc Murici & LB, DW, LV & $\begin{array}{l}\text { understory, sub-canopy, } \\
\text { canopy }\end{array}$ & Neotropical & ES, GO, MG, PE, RJ, RS, SC, SP, BA, CE \\
\hline F. breuteliana Gottsche & EsEc Murici & LB, LV & $\begin{array}{l}\text { understory, sub-canopy, } \\
\text { canopy }\end{array}$ & Neotropical & RJ, PE, RS, SP \\
\hline F. caulisequa (Nees) Nees & EsEc Murici & LB, DW, LV & $\begin{array}{l}\text { understory, sub-canopy, } \\
\text { canopy }\end{array}$ & Neotropical & $\begin{array}{l}\text { AL, AC, BA, CE, ES, MG, PA, PE, RJ, RO, RR, } \\
\text { RS, SC, SE, SP }\end{array}$ \\
\hline F. dusenii Steph. & EsEc Murici & LB & canopy & Neotropical & ES, PE, RJ, RR, RS, SC, SE, SP \\
\hline Frullania ericoides (Nees) Mont. & EsEc Murici & LB, DW & understory & Pantropical & $\begin{array}{l}\text { AC, AM, BA, CE, ES, GO, MG, MS, MT, PA, } \\
\text { PB, PE, PR, RJ, RS, SE, SP }\end{array}$ \\
\hline $\begin{array}{l}\text { F. kunzei (Lehm. \& Lindenb.) } \\
\text { Lehm. \& Lindenb. }\end{array}$ & EsEc Murici & LB, DW, LV & $\begin{array}{l}\text { understory, sub-canopy, } \\
\text { canopy }\end{array}$ & Neotropical & $\begin{array}{l}\text { AC, AM, BA, CE, ES, MG, MT, PA, PB, PE, } \\
\text { PR, RJ, RR, RS, SE, SP }\end{array}$ \\
\hline F. riojaneirensis (Raddi) Ångstr. & EsEc Murici & $\mathrm{LB}, \mathrm{LV}$ & $\begin{array}{l}\text { understory, sub-canopy, } \\
\text { canopy }\end{array}$ & Pantropical & $\begin{array}{l}\text { BA, CE, ES, GO, MG, MS, MT, PA, PB, PE, } \\
\text { PR, RJ, RS, SE, SP }\end{array}$ \\
\hline F. serrata Gottsche & Serra Grande & - & - & Asia, Africa and Brazil & $\mathrm{AL}, \mathrm{RS}, \mathrm{SC}$ \\
\hline \multicolumn{6}{|l|}{ LEJEUNEACEAE } \\
\hline $\begin{array}{l}\text { Acanthocoleus aberrans (Lindenb. \& } \\
\text { Gottsche) Kruijt. }\end{array}$ & Marechal Deodoro & - & - & Africa and America & AL, DF, MG, PR, RS, RJ, SC, SP \\
\hline $\begin{array}{l}\text { Acrolejeunea torulosa (Lehm. \& } \\
\text { Lindenb.) Schiffn. }\end{array}$ & Barra de São Miguel & - & - & Neotropical & $\begin{array}{l}\text { AC, AL, AM, BA, ES, GO, MA, MT, MS, PA, } \\
\text { RJ, RO, RR, SP }\end{array}$ \\
\hline Anoplolejeunea conferta (Meissn.) A. Evans & EsEc Murici & $\mathrm{LB}, \mathrm{LV}$ & $\begin{array}{l}\text { understory, sub-canopy, } \\
\text { canopy }\end{array}$ & Neotropical & BA, ES, MG, PA, PE, RJ, RS, SP \\
\hline
\end{tabular}




\begin{tabular}{|c|c|c|c|c|c|}
\hline Taxa & Locality & Substrata & Forest strata & World distribution & Distribution in Brazil \\
\hline *Aphanolejeunea asperrima Steph. & EsEc Murici & LV & understory & Neotropical & MG, SC \\
\hline *A. camillii (Lehm.) R.M. Schust. & EsEc Murici & LV & understory & Neotropical & $\mathrm{AM}, \mathrm{MG}, \mathrm{PA}, \mathrm{RJ}$ \\
\hline A. truncatifolia Horik. & EsEc Murici & LB, DW, LV & $\begin{array}{l}\text { understory, sub-canopy, } \\
\text { canopy }\end{array}$ & Pantropical & $\begin{array}{l}\text { AM, BA, ES, GO, MG, MS, MT, PA, PE, RJ, } \\
\text { RS, SC, SP }\end{array}$ \\
\hline $\begin{array}{l}\text { Archilejeunea fuscescens (Hampe ex } \\
\text { Lehm.) Fulford }\end{array}$ & $\begin{array}{l}\text { EsEc Murici } \\
\text { and Serra Grande }\end{array}$ & LB & canopy & $\begin{array}{l}\text { South America and } \\
\text { Costa Rica }\end{array}$ & $\mathrm{AC}, \mathrm{AL}, \mathrm{AM}, \mathrm{BA}, \mathrm{PA}, \mathrm{PE}, \mathrm{RJ}, \mathrm{RR}$ \\
\hline A. parviflora (Nees) Schiffn. & EsEc Murici & DW & understory & Neotropical & AC, AM, BA, MG, MS, PA, PE, RJ, RO, RR, RS, SP \\
\hline $\begin{array}{l}\text { Caudalejeunea lehmaniana (Gottsche) } \\
\text { A. Evans }\end{array}$ & $\begin{array}{l}\text { EsEc Murici, } \\
\text { Marechal Deodoro, } \\
\text { and Serra Grande }\end{array}$ & $\mathrm{LB}, \mathrm{LV}$ & understory & Africa and America & $\begin{array}{l}\text { AL, AM, BA, CE, ES, MT, } \\
\text { PA, PE, PR, RS, RO, RR, SC, SE SP }\end{array}$ \\
\hline Ceratolejeunea coarina (Gottsche) Steph. & EsEc Murici & DW, LV & understory & Neotropical & AC, AM, AP, BA, MA, PA PR, SP \\
\hline C. confusa R.M. Schust. & EsEc Murici & LB, DW & $\begin{array}{l}\text { understory, sub-canopy, } \\
\text { canopy }\end{array}$ & $\begin{array}{c}\text { Costa Rica, East } \\
\text { Índias, Chocó, and } \\
\text { Brazil }\end{array}$ & $\mathrm{AM}, \mathrm{PE}, \mathrm{SP}$ \\
\hline C. cornuta (Lindenb.) Schiffn. & $\begin{array}{l}\text { EsEc Murici and } \\
\text { Serra Grande }\end{array}$ & LB, DW, LV & $\begin{array}{l}\text { understory, sub-canopy, } \\
\text { canopy }\end{array}$ & Pantropical & $\begin{array}{l}\text { AC, AL, AM, AP, BA, CE, PA, PE, PR, RJ, RO, } \\
\text { SC, SP }\end{array}$ \\
\hline C. cubensis (Mont.) Schiffn. & EsEc Murici & LB, DW, LV & $\begin{array}{l}\text { understory, sub-canopy, } \\
\text { canopy }\end{array}$ & Neotropical & AC, AM, AP, BA, CE, ES, PA, PB, PE, RJ, RO, SP \\
\hline C. dussiana (Steph.) G. Dauphin & EsEc Murici & LB & understory & Neotropical & $\mathrm{BA}, \mathrm{PA}$ \\
\hline C. falax (Lehm. \& Lindenb.) Bonner & EsEc Murici & LB, LV & $\begin{array}{l}\text { understory, sub-canopy, } \\
\text { canopy }\end{array}$ & Neotropical & AM, MG, PE, RJ, RO, SP \\
\hline C guianensis (Nees \& Mont.) Steph. & $\begin{array}{l}\text { EsEc Murici and } \\
\text { Serra Grande }\end{array}$ & LB, LV & $\begin{array}{l}\text { understory, sub-canopy, } \\
\text { canopy }\end{array}$ & Neotropical & $\mathrm{AM}, \mathrm{AL}, \mathrm{BA}, \mathrm{PA}, \mathrm{PE}$ \\
\hline C. laetefusca (Austin) R.M. Schust. & EsEc Murici & LB, DW, LV & $\begin{array}{l}\text { understory, sub-canopy, } \\
\text { canopy }\end{array}$ & Neotropical & AC, AM, BA, ES, MG, PA, PE, RJ, RR, SP \\
\hline C. minuta Dauphin & EsEc Murici & LB & understory & Brazil and Guianas & $\mathrm{BA}, \mathrm{PA}, \mathrm{PE}$ \\
\hline Cheilolejeunea acutangula (Nees) Grolle & EsEc Murici & LB & understory & Neotropical & AM, BA, ES, GO, MG, MT, PA, PE, RJ, SC, SP \\
\hline C. adnata (Kunze) Grolle & $\begin{array}{l}\text { EsEc Murici and } \\
\text { Serra Grande }\end{array}$ & LB, DW, LV & $\begin{array}{l}\text { understory, sub-canopy, } \\
\text { canopy }\end{array}$ & Neotropical & $\begin{array}{l}\text { AC, AL, AM, BA, ES, MG, MT, PA, PE, PR, RR, } \\
\text { SC, SP }\end{array}$ \\
\hline C. clausa (Nees. \& Mont.) R.M. Schust. & EsEc Murici & LB, DW & $\begin{array}{l}\text { understory, sub-canopy, } \\
\text { canopy }\end{array}$ & Neotropical & $\begin{array}{l}\text { AC, AM, BA, CE, GO, MG, MS, MT, PA, PE, PR, } \\
\text { RJ, SP }\end{array}$ \\
\hline $\begin{array}{l}\text { C. discoidea (Lehm. \& Lindenb.) Kachr. \& } \\
\text { R.M. Schust. }\end{array}$ & EsEc Murici & LB & canopy & Neotropical & BA, ES, MG, MS, MT, RS, SP \\
\hline C. holostipa (Spruce) Grolle \& R.-L. Zhu & EsEc Murici & LB, DW, LV & $\begin{array}{l}\text { understory, sub-canopy, } \\
\text { canopy }\end{array}$ & Neotropical & BA, ES, MG, PA, PR, RJ, SP \\
\hline C. oncophylla (Ångstr.) Grolle \& E. Reiner & EsEc Murici & LB, DW & understory & Neotropical & BA, MG, PR, RJ, RR, SP \\
\hline C. rigidula (Mont.) R.M. Schust. & $\begin{array}{c}\text { EsEc Murici, } \\
\text { Marechal Deodoro, } \\
\text { and Serra Grande }\end{array}$ & LB, DW, LV & $\begin{array}{l}\text { understory, sub-canopy, } \\
\text { canopy }\end{array}$ & Africa and America & $\begin{array}{l}\text { AC, AL, AM, AP, BA, CE, ES, GO, MG, MS, MT, } \\
\text { PA, PB, PE, PR, RJ, RR, SC, SE, SP }\end{array}$ \\
\hline C. trifaria (Reinw. et al.) Mizut. & $\begin{array}{l}\text { EsEc Murici and } \\
\text { Serra Grande }\end{array}$ & LB, DW, LV & $\begin{array}{l}\text { understory, sub-canopy, } \\
\text { canopy }\end{array}$ & Pantropical & $\begin{array}{l}\text { AC, AL, AM, BA, ES, GO, MG, MS, MT, PA, PB, } \\
\text { PE, PR, RJ, RR, SP }\end{array}$ \\
\hline Cololejeunea cardiocarpa (Mont.) A. Evans & EsEc Murici & LV & understory & Pantropical & $\begin{array}{l}\text { AM, BA, ES, GO, MG, MS, MT, PA, PB, PE, PR, } \\
\text { RJ, RO, RR, SC, SP }\end{array}$ \\
\hline${ }^{*}$ C. lanciloba Steph. & EsEc Murici & LV & understory & Pantropical & $\mathrm{AM}$ \\
\hline C. obliqua (Nees \& Mont.) Schiffn. & $\begin{array}{l}\text { EsEc Murici } \\
\text { and Serra Grande }\end{array}$ & LV & understory & Neotropical & AC, AL, AM, PA, PE, PR, RJ, SC, SP \\
\hline
\end{tabular}




\begin{tabular}{|c|c|c|c|c|c|}
\hline Taxa & Locality & Substrata & Forest strata & World distribution & Distribution in Brazil \\
\hline Cololejeunea subcardiocarpa Tixier & $\begin{array}{l}\text { EsEc Murici } \\
\text { and Serra Grande }\end{array}$ & DW, LV & $\begin{array}{l}\text { understory, sub-canopy, } \\
\text { canopy }\end{array}$ & Neotropical & AC, AL, AM, BA, ES, MG, MT, PE, PR, RJ, SC, SP \\
\hline C. submarginata Tixier & Marechal Deodoro & - & - & Neotropical & $\mathrm{AL}, \mathrm{AM}, \mathrm{GO}, \mathrm{MT}$ \\
\hline Colura tortifolia (Nees \& Mont.) Steph. & EsEc Murici & LV & $\begin{array}{l}\text { understory, sub-canopy, } \\
\text { canopy }\end{array}$ & Neotropical & $\mathrm{AC}, \mathrm{CE}, \mathrm{PA}, \mathrm{PE}, \mathrm{RJ}, \mathrm{SP}$ \\
\hline $\begin{array}{l}\text { Cyclolejeunea convexistipa (Lehm. \& } \\
\text { Lindenb.) A. Evans }\end{array}$ & $\begin{array}{l}\text { EsEc Murici } \\
\text { and Serra Grande }\end{array}$ & LB, LV & $\begin{array}{l}\text { understory, sub-canopy, } \\
\text { canopy }\end{array}$ & Neotropical & $\mathrm{AL}, \mathrm{AM}, \mathrm{AP}, \mathrm{BA}, \mathrm{CE}, \mathrm{PA}, \mathrm{SP}$ \\
\hline C. luteola (Spruce) Grolle & EsEc Murici & $\mathrm{LB}, \mathrm{LV}$ & understory & Neotropical & AM, BA, MG, MT, PA, PE, RJ, RR, SP \\
\hline Diplasiolejeunea brunnea Steph. & $\begin{array}{l}\text { EsEc Murici, } \\
\text { Marechal Deodoro, } \\
\text { and Serra Grande }\end{array}$ & LB, LV & $\begin{array}{l}\text { understory, sub-canopy, } \\
\text { canopy }\end{array}$ & Neotropical & AC, AL, AM, BA, CE, MT, PA, RJ, SP \\
\hline D. cavifolia Steph. & EsEc Murici & LB, LV & understory, sub-canopy, canopy & Pantropical & CE, PE, PR, SC, SP \\
\hline D. cobrensis Gottsche ex Steph. & Marechal Deodoro & - & - & Pantropical & AL, BA, PE, RO \\
\hline D. latipuensis Tixier & EsEc Murici & LV & understory & Guiana and Brazil & $\mathrm{BA}, \mathrm{SP}$ \\
\hline D. pellucida (Meissn.) Schiffn. & Murici and Serra Grande & de LV & understory, sub-canopy, canopy & y Neotropical & $\mathrm{AL}, \mathrm{AM}, \mathrm{BA}, \mathrm{PA}, \mathrm{RJ}, \mathrm{SC}, \mathrm{SP}$ \\
\hline D. rudolphiana Steph. & EsEc Murici & LB, LV & understory, sub-canopy, canopy & Pantropical & AC, AM, BA, ES, PB, PE, RJ, SC, SE, SP \\
\hline Drepanolejeunea bidens (Steph.) A. Evans & Serra Grande & - & - & Neotropical & $\mathrm{AL}, \mathrm{AM}, \mathrm{MG}, \mathrm{PB}, \mathrm{PE}, \mathrm{RR}, \mathrm{SP}$ \\
\hline *D. campanulata (Spruce) Steph. & EsEc Murici & LB, LV & understory, sub-canopy, canopy & $\begin{array}{l}\text { North Andes and } \\
\text { Southeastern Brazil }\end{array}$ & $\mathrm{RJ}, \mathrm{SC}, \mathrm{SP}$ \\
\hline$* D$. crucianella (Tayl.) A. Evans & EsEc Murici & LV & understory & Neotropical & $\mathrm{AM}, \mathrm{PA}$ \\
\hline D. fragilis Bischl. & EsEc Murici & LB, DW, LV & $\begin{array}{l}\text { understory, sub-canopy, } \\
\text { canopy }\end{array}$ & Neotropical & AM, BA, ES, PA, PE, RJ, RR, SP \\
\hline D. mosenii (Steph.) Bischl. & EsEc Murici & LB, LV & understory, sub-canopy, canopy & Neotropical & AM, BA, ES, MS, PE, PR, RJ, RS, SC, SP \\
\hline $\begin{array}{l}\text { Frullanoides corticalis (Lehm. \& Lindenb.) } \\
\quad \text { van Slageren }\end{array}$ & EsEc Murici & LB & understory, canopy & Neotropical & BA, MG, MT, RJ, RR, SP \\
\hline Harpalejeunea oxyphylla (Nees \& Mont.) Steph. & ph. EsEc Murici & DW, LV & understory & Neotropical & AM, BA, PA, PB, PE, RJ, SP \\
\hline H. stricta (Lindenb. \& Gottsche) Steph. & EsEc Murici & LB, DW, LV & $\begin{array}{l}\text { understory, sub-canopy, } \\
\text { canopy }\end{array}$ & Neotropical & $\mathrm{AC}, \mathrm{BA}, \mathrm{PA}, \mathrm{PE}, \mathrm{RJ}, \mathrm{SP}$ \\
\hline$*$ H. tridens (Besch. \& Spruce) Steph. & EsEc Murici & LB, DW & $\begin{array}{l}\text { understory, sub-canopy, } \\
\text { canopy }\end{array}$ & $\begin{array}{l}\text { East Índias, } \\
\text { South America } \\
\text { and Brazil }\end{array}$ & SP \\
\hline Lejeunea cancellata Nees \& Mont. & Serra Grande & - & C & $\begin{array}{l}\text { Cuba, USA, Argentina } \\
\text { and Brazil }\end{array}$ & AL, BA, CE, AS, MS, MT, RJ, SC, SP \\
\hline L. caulicalyx (Steph.) E. Reiner \& Goda & EsEc Murici & LB, DW & understory & Neotropical & AC, BA, CE, ES, MS, MT, PA, PE, PR, RJ, RR, SP \\
\hline L. cerina (Lehm. \& Lindenb.) Gottsche & EsEc Murici & LB & understory, canopy & Neotropical & AC, ES, PE, RJ, SP \\
\hline L. controversa Gottsche & EsEc Murici & LB, DW & understory & Neotropical & AC, MS, PA, SP \\
\hline L. elliotti Spruce & EsEc Murici & LB, DW & understory & Neotropical & BA \\
\hline L. filipes Spruce & EsEc Murici & $\mathrm{LB}, \mathrm{LV}$ & $\begin{array}{l}\text { understory, sub-canopy, } \\
\text { canopy }\end{array}$ & $\begin{array}{c}\text { Dominica, Peru, } \\
\text { Argentina, and } \\
\text { Brazil }\end{array}$ & BA, MS, SP \\
\hline L. flava (Sw.) Nees & EsEc Murici & LB, DW, LV & $\begin{array}{l}\text { understory, sub-canopy, } \\
\text { canopy }\end{array}$ & Pantropical & $\begin{array}{l}\text { AC, AM, BA, CE, ES, GO, MG, MS, MT, PA, } \\
\text { PB, PE, PR, RJ, RR, RS, SP }\end{array}$ \\
\hline L. grossiretis (Steph.) E. Reiner \& Goda & EsEc Murici & DW & understory & Brazil & BA, MG, RJ, SP \\
\hline
\end{tabular}


Table 1 (continuation)

\begin{tabular}{|c|c|c|c|c|c|}
\hline Taxa & Locality & Substrata & Forest strata & World distribution & Distribution in Brazil \\
\hline Lejeunea grossitexta (Steph.) E. Reiner \& God & oda EsEc Murici & DW, LV & understory & $\begin{array}{l}\text { Brasil, Paraguai, and } \\
\text { northern Argentina }\end{array}$ & ES, MG, PE, PR, RJ, SC, SP \\
\hline L. immersa Spruce & EsEc Murici & LV & understory & Neotropical & $\mathrm{AM}, \mathrm{BA}, \mathrm{PA}, \mathrm{PE}$ \\
\hline L. laetevirens Nees \& Mont. & $\begin{array}{l}\text { Barra de São Miguel, } \\
\text { EsEc Murici, Maceió, } \\
\text { enedo, and Serra Grande }\end{array}$ & LB, DW, LV & $\begin{array}{l}\text { understory, sub-canopy, } \\
\text { canopy }\end{array}$ & Neotropical & $\begin{array}{l}\text { AC, AL, AM, BA, CE, ES, MS, MT, PA, PB, } \\
\text { PE, RJ, RR, SP }\end{array}$ \\
\hline L. magnolia Lindenb. \& Gottsche & $\begin{array}{l}\text { EsEc Murici and } \\
\text { Serra Grande }\end{array}$ & LB, DW, LV & $\begin{array}{l}\text { understory, sub-canopy, } \\
\text { canopy }\end{array}$ & Africa and America & AC, AL, BA, CE, ES, MS, MT, PA, PE, RJ, SP \\
\hline L. phyllobola Nees \& Motn. & EsEc Murici and Penedo & LB, DW & $\begin{array}{l}\text { understory, sub-canopy, } \\
\text { canopy }\end{array}$ & Africa and America & AC, AL, AM, BA, CE, ES, MS, PA, RJ, RS, SP \\
\hline L. puiggariana Steph. & EsEc Murici & $\mathrm{LB}, \mathrm{LV}$ & $\begin{array}{l}\text { understory, sub-canopy, } \\
\text { canopy }\end{array}$ & $\begin{array}{l}\text { Brazil and } \\
\text { northern Argentina }\end{array}$ & MS, MT, PE, SP \\
\hline L. tapajosensis Spruce & EsEc Murici & LB, DW & understory & Brazil and Argentina & AC, BA, ES, PA, PE \\
\hline Lepidolejeunea involuta (Gottsche) Grolle & EsEc Murici & LB, LV & understory, canopy & Neotropical & AM, ES, PA, PE, PR, RO, RR, SP \\
\hline $\begin{array}{l}\text { Leptolejeunea elliptica (Lehm. \& Lindeb.) } \\
\text { Schiffn. }\end{array}$ & $\begin{array}{l}\text { EsEc Murici and } \\
\text { Serra Grande }\end{array}$ & LB, LV & $\begin{array}{l}\text { understory, sub-canopy, } \\
\text { canopy }\end{array}$ & Neotropical & AL, BA, ES, MG, PE, RJ, RS, SC, SP \\
\hline $\begin{array}{l}\text { Leucolejeunea xanthocarpa (Lehm. \& } \\
\text { Lindenb.) A. Evans }\end{array}$ & EsEc Murici & LB, DW, LV & $\begin{array}{l}\text { understory, sub-canopy, } \\
\text { canopy }\end{array}$ & Pantropical & BA, ES, MG, PE, RJ, RS, SC, SP \\
\hline Lopholejeunea subfusca (Nees) Schiffn. & EsEc Murici & LB, DW, LV & $\begin{array}{l}\text { understory, sub-canopy, } \\
\text { canopy }\end{array}$ & Pantropical & $\begin{array}{l}\text { AC, AM, BA, CE, ES, MG, MS, MT, PA, PB, } \\
\text { PE, RJ, RO, RR, SC, SP }\end{array}$ \\
\hline Microlejeunea epiphylla Bischl. & EsEc Murici & $\mathrm{LB}, \mathrm{LV}$ & $\begin{array}{l}\text { understory, sub-canopy, } \\
\text { canopy }\end{array}$ & Neotropical & AP, BA, ES, MG, MS, PA, PB, PE, SP \\
\hline Neurolejeunea breutelli (Gottsche) A. Evans & EsEc Murici & LV & understory & $\begin{array}{c}\text { Neotropics and North } \\
\text { America }\end{array}$ & BA, ES, MG, PA, PE, RJ, RS, SC, SP \\
\hline Odontolejeunea lunulata (Weber) Schiffn. & EsEc Murici & LV & canopy & Neotropics and East Africa & $\begin{array}{l}\text { AC, AP, AM, BA, CE, ES, MG, MT, PA, PE, } \\
\text { PR, RR, RS, SP }\end{array}$ \\
\hline Prionolejeunea denticulata (Weber) Schiffn. & EsEc Murici & LB, DW, LV & $\begin{array}{l}\text { understory, sub-canopy, } \\
\text { canopy }\end{array}$ & Neotropical & $\mathrm{AM}, \mathrm{CE}, \mathrm{PA}, \mathrm{PE}, \mathrm{RJ}$ \\
\hline Pycnolejeunea contigua (Nees) Grolle & EsEc Murici & LB, LV & $\begin{array}{l}\text { understory, sub-canopy, } \\
\text { canopy }\end{array}$ & Pantropical & AM, BA, CE, ES, ES, MG, PA, PE, RR, RS, SC, SP \\
\hline P. macroloba (Nees \& Mont.) Schiffn. & Ec Murici and Junqueiro & LB & understory & Neotropical & $\mathrm{AL}, \mathrm{AM}, \mathrm{BA}, \mathrm{CE}, \mathrm{ES}, \mathrm{PA}, \mathrm{PE}, \mathrm{SP}$ \\
\hline $\begin{array}{l}\text { Rectolejeunea berteroana (Gottsche ex } \\
\text { Steph.) A. Evans }\end{array}$ & EsEc Murici $\quad \mathrm{L}$ & LB, DW, LV & $\begin{array}{l}\text { understory, sub-canopy, } \\
\text { canopy }\end{array}$ & Neotropical & AC, AM, BA, ES, PA, PE, PR, RJ, SC, SP \\
\hline R. emarginuliflora (Gottsche) A. Evans & EsEc Murici & LB & understory & Neotropical & BA, SP \\
\hline R. flageliformis A. Evans & EsEc Murici & DW, LV & understory & Neotropical & $\mathrm{BA}, \mathrm{PE}, \mathrm{SP}$ \\
\hline Schiffneriolejeunea polycarpa (Nees) Gradst. & $\begin{array}{l}\text { EsEc Murici and } \\
\text { Serra Grande }\end{array}$ & LB & canopy & Pantropical & $\begin{array}{l}\text { AC, AL, AM, BA, CE, ES, GO, MG, MS, MT, } \\
\text { PA, PB, PE, RJ, RR, RS, SC, SE, SP }\end{array}$ \\
\hline $\begin{array}{l}\text { Stictolejeunea squamata (Willd. ex Weber) } \\
\text { Schiffn. }\end{array}$ & $\begin{array}{l}\text { EsEc Murici and } \\
\text { Serra Grande }\end{array}$ & LB, DW & understory & Neotropical & AC, AL, AM, AP, BA, ES, MG, PA, PE, RJ, RS, SP \\
\hline $\begin{array}{l}\text { Symbiezidium barbiflorum (Lindenb. \& } \\
\text { Gottsche) A. Evans }\end{array}$ & $\begin{array}{l}\text { EsEc Murici and } \\
\text { Serra Grande }\end{array}$ & LB, DW, LV & $\begin{array}{l}\text { understory, sub-canopy, } \\
\text { canopy }\end{array}$ & Neotropical & AC, AL, AM, BA, ES, PA, PE, RJ, SC, SP \\
\hline Taxilejeunea obtusangula (Spruce) A. Evans & Serra Grande & - & - & Neotropical & $\mathrm{AL}, \mathrm{AM}, \mathrm{RR}$ \\
\hline $\begin{array}{l}\text { Vitalianthus bischlerianus (Pôrto \& Grolle) } \\
\text { R.M. Schust. \& Giancotti }\end{array}$ & EsEc Murici & $\mathrm{LB}, \mathrm{LV}$ & understory & Neotropical & BA, PE, PR, RJ, SC, SP \\
\hline
\end{tabular}

L. tapajosensis Spruce

Lepidolejeunea involuta (Gottsche) Grolle Schiff

Lindenb.) A. Evan

ejeunea subfusca (Nees) Schiffn.

EsEc Murici

Neurolejeunea breutelli (Gottsche) A. Evans

ics and North

As and East Afric

, PA, PE

RR, RS, SP

M, BA, CE, ES, ES, MG, PA, PE, RR, RS, SC, SP

L, AM, BA, CE, ES, PA, PE, SP

A, SP

BA, PE, SP

R, RS, SC, SE, SP

Serc Murici and

$$
\text { canopy }
$$

R.M. Schust. \& Giancott 


\begin{tabular}{|c|c|c|c|c|c|}
\hline Taxa & Locality & Substrata & Forest strata & World distribution & Distribution in Brazil \\
\hline $\begin{array}{l}\text { Xylolejeunea crenata (Nees \& Mont.) } \\
\text { X.-L. He \& Grolle }\end{array}$ & EsEc Murici & LB, DW & understory & Neotropical & AM, BA, MA, MG, PA, PE, RJ, RO, RR, SP \\
\hline \multicolumn{6}{|l|}{ LEPIDOZIACEAE } \\
\hline Arachniopsis diacantha (Mont.) Howe & EsEc Murici & LB, DW & understory & Pantropical & AC, AM, BA, ES, PA, PE, PR, RJ, RS, SP \\
\hline Bazzania heterostipa (Steph.) Fulford & EsEc Murici & DW & understory & Brazil & ES, MG, PE, PR, RJ, RS, SC, SP \\
\hline \multicolumn{6}{|l|}{ METZGERIACEAE } \\
\hline Metzgeria albinea Spruce & EsEc Murici & DW, LV & understory & Pantropical & BA, CE, ES, MG, PA, PE, PR, RJ, RS, SC, SP \\
\hline M. conjugata Lindb. & EsEc Murici & LB & canopy & Subcomsmopolitan & CE, ES, MG, PE, PR, RJ, RS, SP \\
\hline M. dichotoma (Sw.) Nees & EsEc Murici and Serra Grande & LV & understory & Neotropical & $\mathrm{AL}, \mathrm{GO}, \mathrm{MG}, \mathrm{PB}, \mathrm{PE}, \mathrm{RJ}, \mathrm{RS}, \mathrm{SP}$ \\
\hline M. myriopoda Lindb. & EsEc Murici & $\mathrm{LV}$ & understory & Neotropics and North America & $\mathrm{ES}, \mathrm{PE}, \mathrm{MG}, \mathrm{RJ}, \mathrm{RS}, \mathrm{SC}, \mathrm{SP}$ \\
\hline \multicolumn{6}{|l|}{ PLAGIOCHILACEAE } \\
\hline $\begin{array}{l}\text { Plagiochila disticha (Lehm. \& } \\
\text { Lindenb.) Lindenb. }\end{array}$ & $\begin{array}{l}\text { EsEc Murici and } \\
\text { Serra Grande }\end{array}$ & LB, DW, LV & $\begin{array}{l}\text { understory, sub-canopy, } \\
\text { canopy }\end{array}$ & Neotropical & $\begin{array}{l}\text { AC, AL, AM, AP, CE, DF, ES, MG, MS, MT, } \\
\text { PA, PB, PE, RJ, RR, RS, SC, SP }\end{array}$ \\
\hline P. distinctifolia Lindenb. & EsEc Murici & LB & canopy & Neotropical & AM, PA, PE, MG, RJ \\
\hline P. gymnocalycina (Lehm. \& Lindenb.) & Lindenb. EsEc Murici & LB & understory & Neotropical & $\mathrm{AC}, \mathrm{MG}, \mathrm{PE}, \mathrm{RJ}, \mathrm{SC}, \mathrm{SP}$ \\
\hline P. martiana (Nees) Lindenb. & $\begin{array}{l}\text { EsEc Murici and } \\
\text { Serra Grande }\end{array}$ & LB, DW, LV & $\begin{array}{l}\text { understory, sub-canopy, } \\
\text { canopy }\end{array}$ & Neotropical & $\begin{array}{l}\text { AC, AL, CE, DF, ES, MG, MS, MT, PE, PR, RJ, } \\
\text { RS, SC, SP }\end{array}$ \\
\hline P. montagnei Nees & EsEc Murici & LB, DW & $\begin{array}{l}\text { understory, sub-canopy, } \\
\text { canopy }\end{array}$ & Neotropical & $\mathrm{AC}, \mathrm{AM}, \mathrm{AP}, \mathrm{BA}, \mathrm{ES}, \mathrm{PA}, \mathrm{PE}, \mathrm{PR}, \mathrm{RJ}, \mathrm{SP}$ \\
\hline \multicolumn{6}{|l|}{ RADULACEAE } \\
\hline Radula flaccida Lindenb. \& Gottsche & EsEc Murici & LV & understory & Africa and America & AC, AM, BA, MG, PA, PR, RR, SP \\
\hline R. kegelii Gottsche ex Steph. & EsEc Murici & LB, DW & understory & Neotropical & $\begin{array}{l}\text { AL, AM, AP, BA, BA, ES, MG, MT, PA, PR, } \\
\text { RJ, RS, SC, SP }\end{array}$ \\
\hline R. ligula Steph. & EsEc Murici & DW & understory & Brazil and Argentina & PR, RJ, RS, SC \\
\hline R. recubans Tayl. & $\begin{array}{l}\text { EsEc Murici, Marechal } \\
\text { Deodoro and Serra Grande }\end{array}$ & LB, DW & understory & $\begin{array}{c}\text { Neotropics and subtropical } \\
\text { America }\end{array}$ & AC, AL, PA, PE, RJ, RS, SP \\
\hline \multicolumn{6}{|l|}{ RICCIACEAE } \\
\hline Riccia vitalii Jovet-Ast. & Arapiraca & - & - & Costa Rica and Brazil & $\begin{array}{l}\text { AL, AM, BA, CE, ES, GO, MA, MS, PB, PE, } \\
\text { PI, RN, SE, TO }\end{array}$ \\
\hline
\end{tabular}


Description: Gradstein \& Costa (2003).

Comments: this species is characterized by well developed lobules in most leaves, reaching $2 / 5$ to $2 / 3$ of lobe length and with the first lobule tooth falcate, consisting of two slightly elongated cells; the surface of lobes, lobules, and perianths is covered with conical papillae. The marginal cells of lobes and lobules are conically protuberant. Aphanolejeunea asperrima resembles A. microscopica (Tayl.) A. Evans, but the latter has greater lobules that reach $2 / 3$ to $4 / 5$ of lobe length. Aphanolejeunea asperrima has only been recorded for Patagonia and Brazil, where it has been found growing on leaves at altitudes that vary from 1450-2000 m. In Brazil it was previously known to the South and Southeastern regions. It was found growing on leaves in the understory at EsEc Murici.

Aphanolejeunea camillii (Lehm.) R.M. Schust., Hepat. Anthocer. N. Amer. 4: 1297. 1980.

Fig. 7-10

Material examined: BRAZIL. Alagoas: Murici, 2/XII/2004, Pôrto (UFP 45844); ib. 2/XII/2004, Pôrto (UFP 45827).

Description: Gradstein \& Costa (2003).

Comments: this species is characterized by elliptic, rhombic, or lanceolate leaves that are wider in the middle, nearly symmetrical, and with an acute apex that ends in one cell. The lobules are reduced in most leaves, but are occasionally developed and can reach $1 / 4$ of lobe length. Aphanolejeunea camillii resembles A. paucifolia (Spruce) E. Reiner, but it is clearly distinguished by the leaves - those of the latter are asymmetrical, wider at the base, and with an apex that ends in a row of 3-4 cells; in addition, the lobules are always reduced. It is common in the Neotropics, occurring from Mexico to Paraguay and northern Argentina. In Brazil it is known to occur in the North and Southeast regions. It was found growing on leaves in the understory in the EsEc Murici.

Cololejeunea lanciloba Steph., Hedwigia 34: 250. 1895. Fig. 11-16

Material examined: BRAZIL. Alagoas: Murici, 3/XII/2004, Pôrto (UFP 45828).

Description: Tixier (1991), as Cololejeunea evansii P. Tixier.

Comments: this species is characterized by leaves with a hyaline margin of rectangular cells and with plane lobules parallel to the stem frequently bilobate and with a rounded apex. Cololejeunea lanciloba has highly variable gametophytic characters, even concerning lobule shape; the latter fact causes problems in distinguishing it from Cololejeunea latilobula (Herzog) Tixier, according Dr. Tamás Pócs who is presently revising the genera for the Neotropics (pers. comm.). The only reliable distinction between them is that $C$. lanciloba has narrowly ligulate to acute lobules usually with 1-2 lateral teeth while C. latilobula always has broad, obtuse, ligulate lobules. Cololejeunea lanciloba is Pantropical and grows on leaves in areas with altitudes up to $50 \mathrm{~m}$. In Brazil it was restricted to Amazonas state, where it was recorded by the first name, Cololejeunea tonkinensis Steph. It was found growing on leaves in the understory at EsEc Murici.

Drepanolejeunea campanulata (Spruce) Steph., Sp. Hepat. 5: 328. 1913.

Fig. 17-21

Material examined: BRAZIL. Alagoas: Murici, 2/XII/2004, Pôrto (UFP 45826).

Description: Bischler (1964).

Comments: this species is characterized by the presence of a conspicuous tooth 2-3 cells long on the ventral lobe margin, positioned just above the junction with the keel. This species resembles Drepanolejeunea lichenicola (Spruce) Steph., but the pre-apical lobule teeth of the latter are longer, curved, and usually cross the apical teeth, whereas in D. campanulata the teeth are short and inconspicuous. Drepanolejeunea campanulata was previously known to Ecuador, Peru, and Southeast Brazil. It was found growing on leaves and bark in the understory, sub-canopy, and canopy at EsEc Murici.

Drepanolejeunea crucianella (Tayl.) A. Evans, Bull. Torrey Bot. Club 30: 33. 1903.

Fig. 22-26

Material examined: BRAZIL. Alagoas: Murici, 2/XII/2004, Pôrto (UFP 45822); ib. 2/XII/2004, Pôrto (UFP 45824); ib. 2/XII/2004, Pôrto (UFP 45833); ib. 2/XII/2004, Pôrto (UFP 45841); ib. 2/XII/2004, Pôrto (UFP 45845).

Description: Bischler (1964).

Comments: this species can be easily recognized due to the presence of conspicuous teeth that are 2-6 cells long and 1-3 cells wide at the base in both the dorsal and ventral leaf margins. This species resembles Drepanolejeunea palmifolia (Nees) Steph., but the latter has teeth only on dorsal margins. Drepanolejeunea crucianella generally occurs in lowland forests, growing on leaves. It has a Neotropical distribution and in Brazil it was previously restricted to Amazonas and Pará, in the North region. It was found growing on leaves in the understory at EsEc Murci.

Harpalejeunea tridens (Besch. \& Spruce) Steph., Sp. Hepat. 5: 263. 1913.

Fig. 27-30 

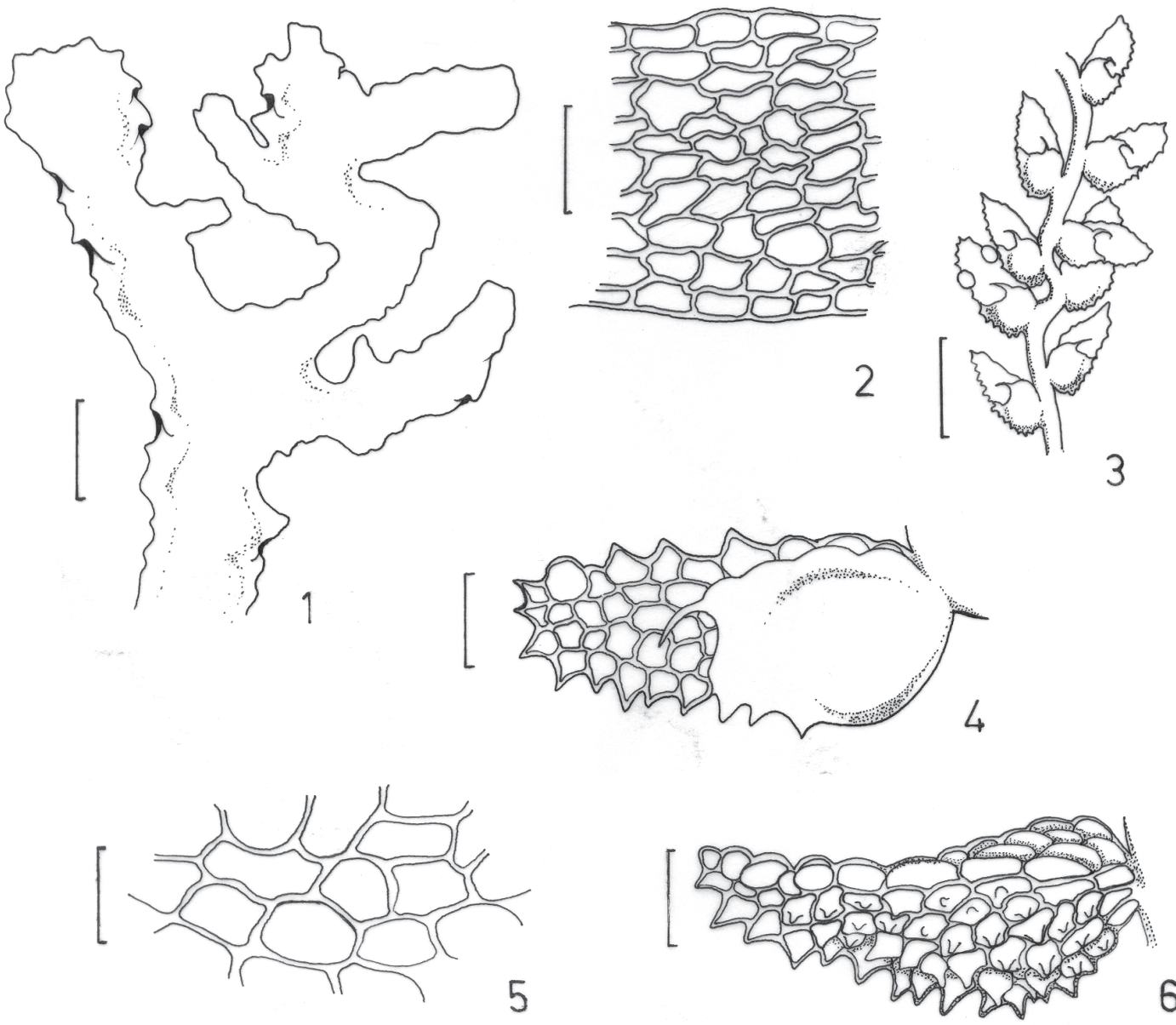

5
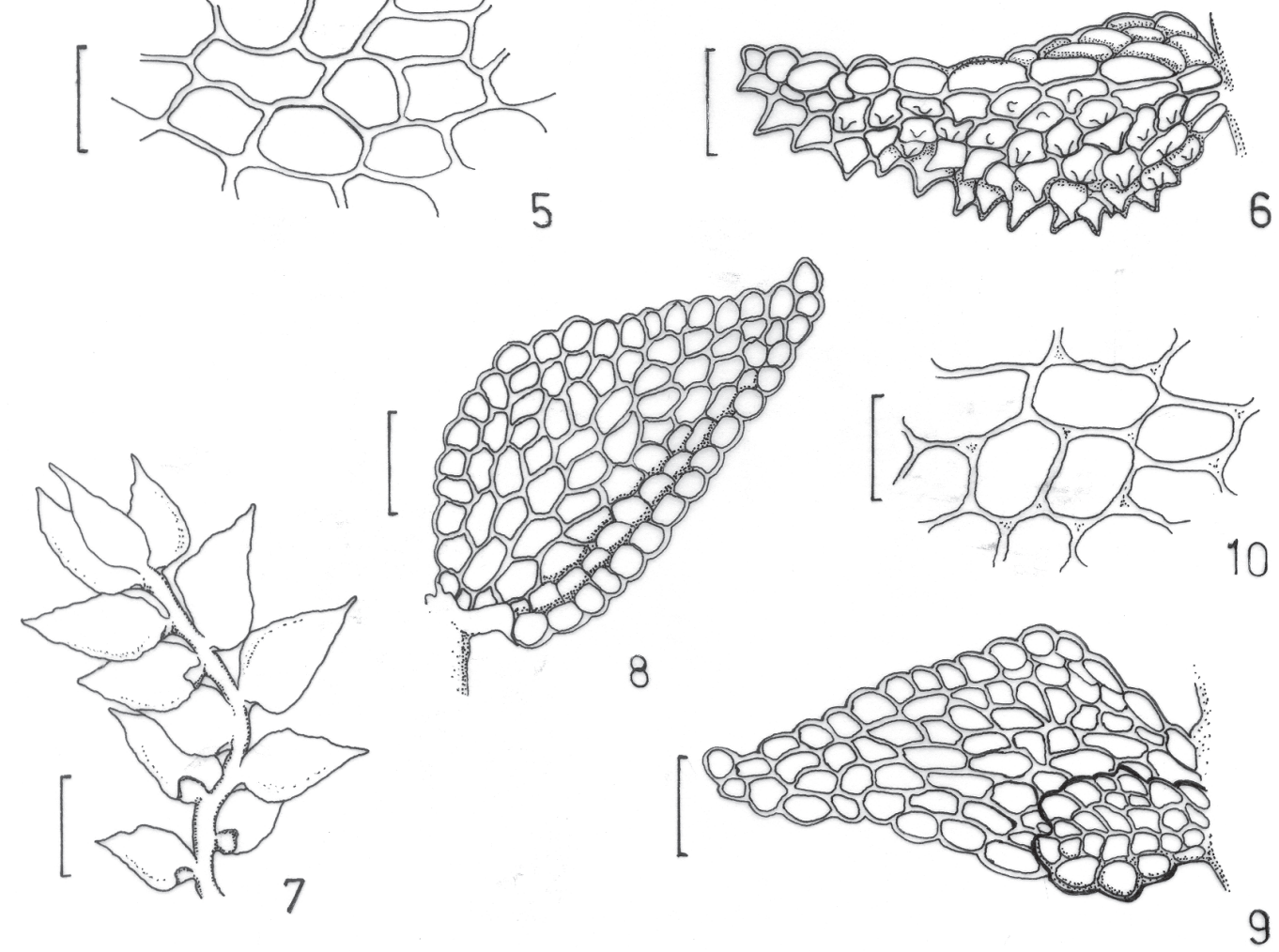

Figures 1-10. 1-2. Aneura pinguis (L.) Dumort. 1. Habit, dorsal view. 2. Cross section of main axis. Figures 3-6. Aphanolejeunea asperrima Steph. 3. Habit, ventral view. 4, 6. Leaves. 5. Median cells of leaf. Figures 7-10. Aphanolejeunea camillii (Lehm.) R.M. Schust. 7. Habit,

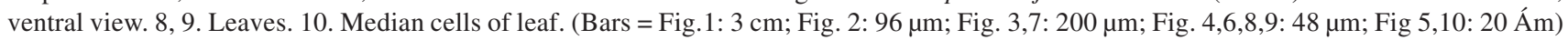



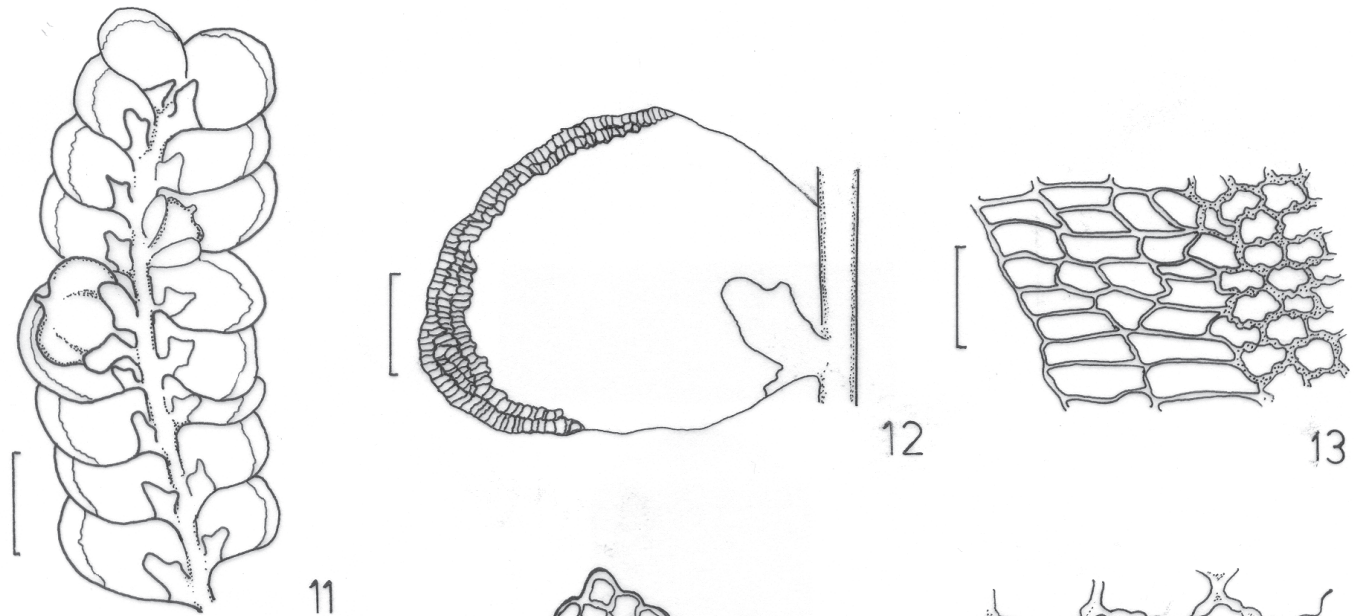

11
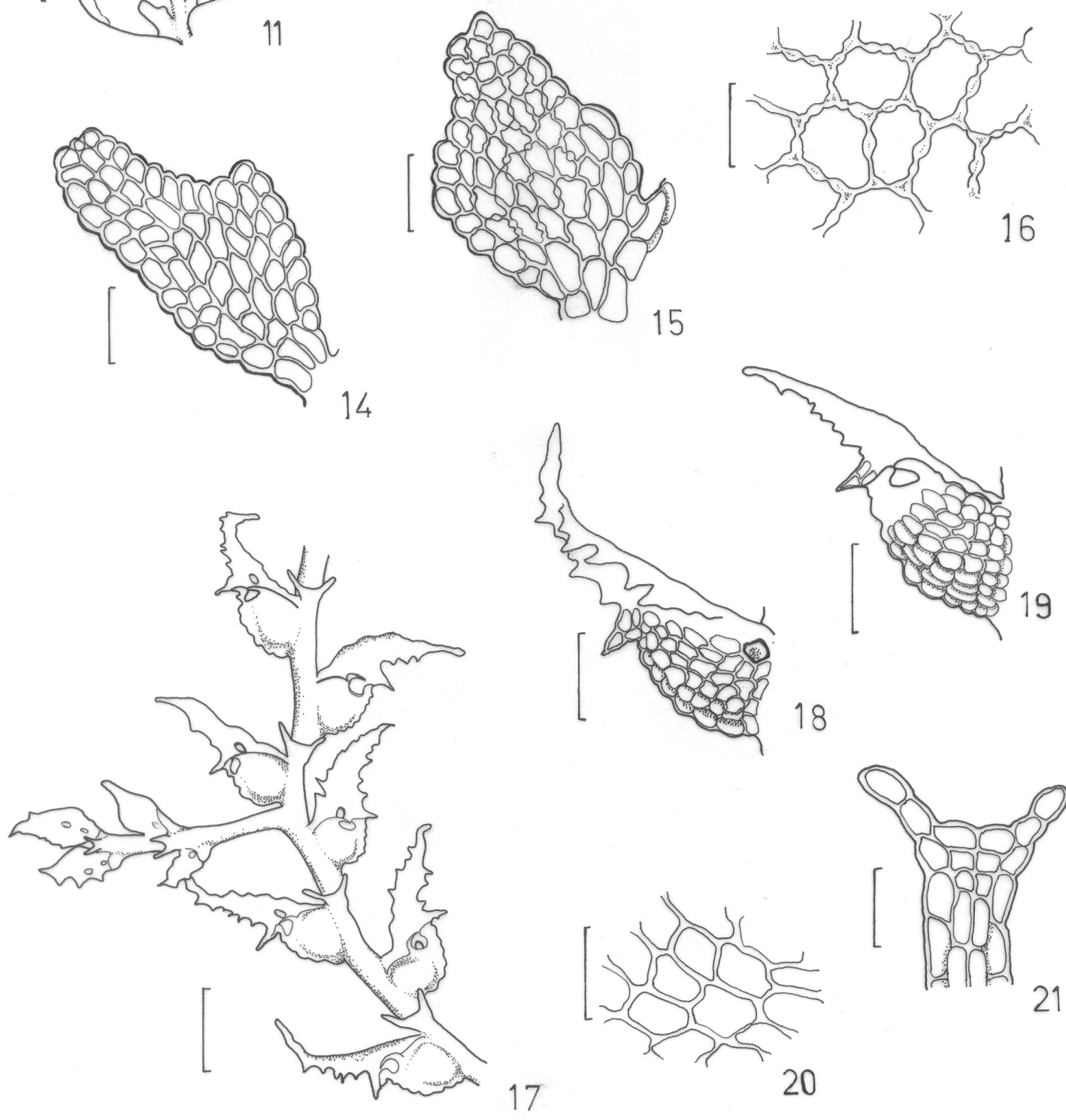

17
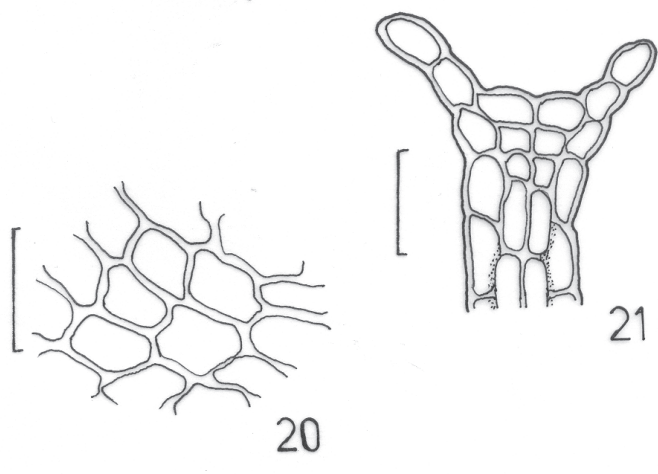

Figures 11-21. 11-16.Cololejeunea lanciloba Steph. 11. Habit, ventral view. 12. Leaf, ventral view. 13. Marginal cells of leaf. 14, 15. Lobules. 16. Median cells of leaf. Figures 17-21. Drepanolejeunea campanulata (Spruce) Steph. 17. Habit, ventral view. 18. Leaf, dorsal view. 19. Leaf,

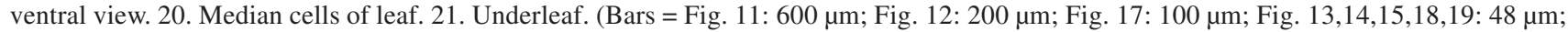
Fig. 21: $30 \mu \mathrm{m}$; Fig. 16,20: $20 \mu \mathrm{m}$ ). 

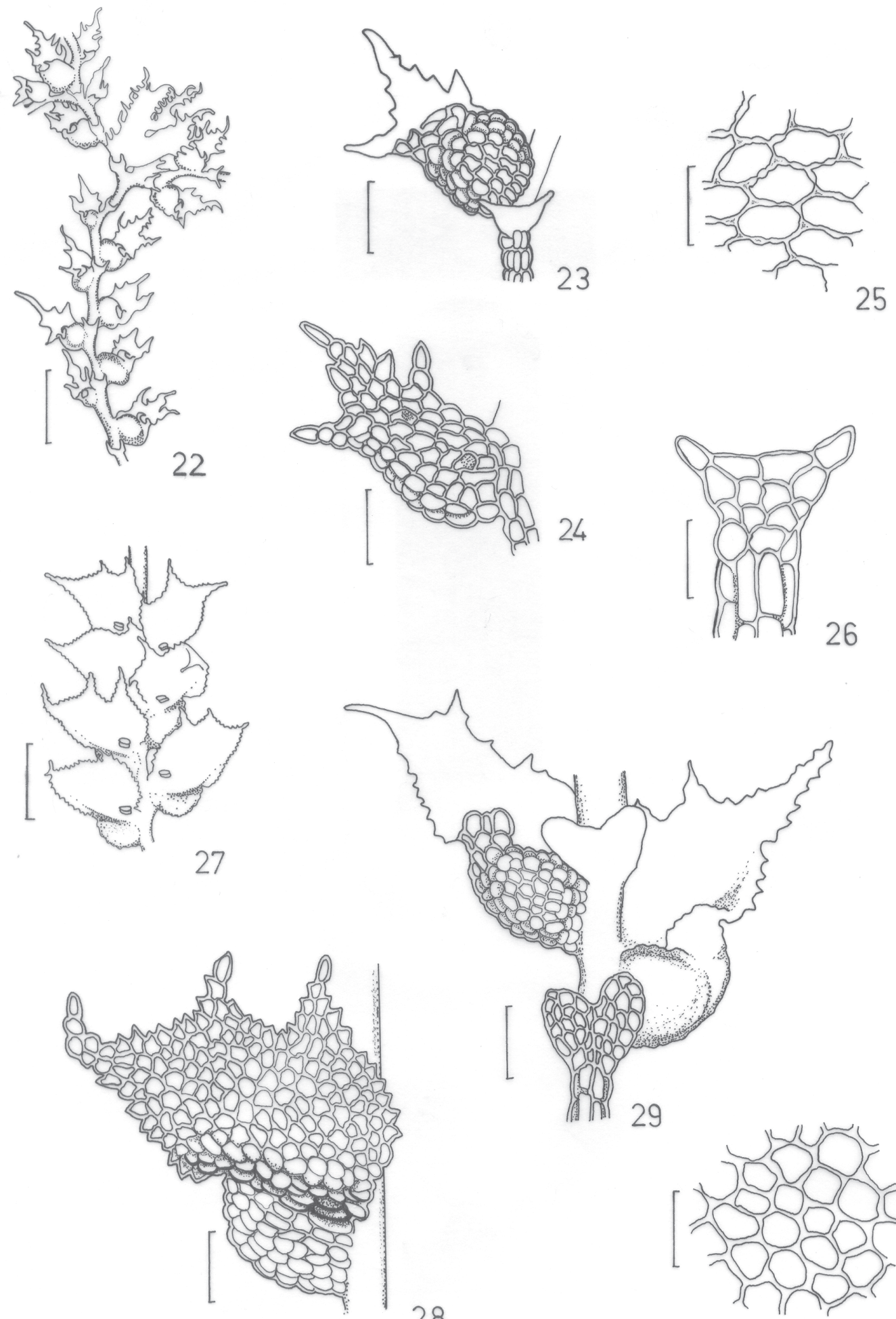

28
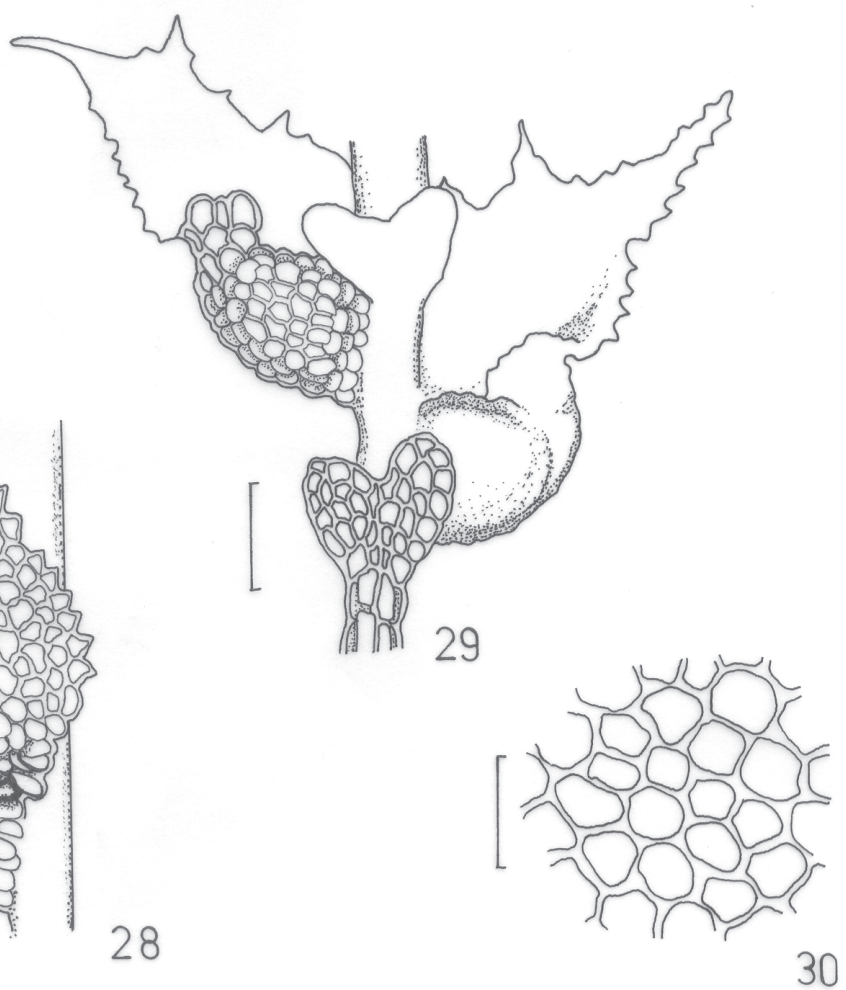

Figures 22-30. 22-26. Drepanolejeunea crucianella (Tayl.) A. Evans. 22. Habit, ventral view. 23. Leaf, ventral view. 24. Leaf, dorsal view. 25. Median cells of leaf. 26. Underleaf. Figures 27-30. Harpalejeunea tridens (Besch. \& Spruce) Steph. 27. Habit, ventral view. 28. Leaf, dorsal view. 29. Detailed gametophyte, ventral view. 30. Median cells of leaf. (Bars: Fig. 22: $200 \mu$ m; Fig. 23,24,28,29: 48 $\mu$ m; Fig.25,26,30: $20 \mu \mathrm{m}$; Fig. 27: $168 \mu \mathrm{m}$ ). 
Material examined: BRAZIL. Alagoas: Murici, 2/XII/2004, Pôrto (UFP 45849); ib. 25/III/2006, Alvarenga (UFP 45846); ib. 25/III/2006, Alvarenga (UFP 45847); ib. 25/III/2006, Alvarenga (UFP 45848).

Description: Evans (1902), as Harpalejeunea heterodonta A. Evans.

Comments: this species has leaf margins typically crenate, with an acute to piliferous apex that ends in a row of 3-4 cells; there is also one or more additional teeth along the dorsal margin. This species was found growing on bark and leaves in lowland rain forests. In Brazil it was previously referred only to São Paulo, in the Southeast region. It occurred on bark and decaying wood in the understory, sub-canopy and canopy at EsEc Murici.

\section{Acknowledgements}

The authors would like to thank the following specialists for help in specimen identification: Dr. Anna Luiza Ilkiu-Borges (Museu Paraense Emílio Goeldi); Dr. Denise Pinheiro da Costa (Jardim Botânico do Rio de Janeiro); Dr. Alfons Schäfer-Verwimp, Dr. Elena ReinerDrehwald, and Dr. Stephan Robert Gradstein (Göttingen University, Germany); Dr. Támas Pócs (Ezsterhazy Teachers' College, Hungary); and Dr. Olga Yano (Instituto de Botânica de São Paulo). We would also like to thank the Fundação O Boticário de Proteção à Natureza and CNPq for financial support.

\section{References}

Bastos, C.J.P. \& Yano, O. 2004. New records of Lejeuneaceae (Marchantiophyta) for the Brazil. Acta Botanica Malacitana 29: $13-21$.

Bastos, C.J.P. \& Yano, O. 2006. Briófitas de restinga das regiões Metropolitana de Salvador e Litoral Norte do Estado da Bahia, Brasil. Boletim do Instituto de Botânica 18: 198-205.

Bischler, H. 1964. Le genre Drepanolejeunea Steph. en Amérique Centrale et Méridionale. Revue Briologique et Lichénoloque 33: $15-133$

Brasil - MMA. 2002. Avaliação e identificação de áreas e ações prioritárias para a conservação, utilização sustentável e repartição dos benefícios da biodiversidade nos biomas brasileiros. Brasília, Ministério do Meio Ambiente/SBF.

Câmara, P.E.A.S. \& Vital, D.M. 2004. Briófitas do Municipio de Poconé, Pantanal de Mato Grosso, MT, Brasil. Acta Botanica Brasilica 18: $881-886$

Câmara, P.E.A.S. \& Costa, D.P. 2006. Hepáticas e antóceros das matas de galeria da Reserva Ecológica do IBGE, RECOR, Distrito Federal, Brasil. Hoehnea 33: 79-87.

Costa, D.P. \& Silva, A.G. 2003. Briófitas da Reserva Natural do Rio Doce, Linhares, Espírito Santo, Brasil. Boletim do Museu de Biologia Mello Leitão, Nova Série, 16: 21-38.

Costa, D.P.; Imbassahy, C.A.A. \& Silva, V.P.A.V. 2005. Checklist and distribution of mosses, liverworts and hornworts of Rio de Janeiro state, Brazil. The Journal of the Hattori Botanical Laboratory 98: 259-298.

Crandall-Stotler, B. \& Stotler, R.E. 2000. Morphology and classification of the Marchantiophyta. Pp. 21-70. In: A.J. Shaw \& B. Goffinet (orgs.) Bryophyte Biology. Cambridge, Cambridge University Press.
Dauphin, G. 2003. Ceratolejeunea. Flora Neotropica, monograph 90: $1-86$.

Evans, A. 1902. Hepaticae of Puerto Rico. III. Harpalejeunea, Cyrtolejeunea, Euosmolejeunea and Trachylejeunea. Bulletin of the Torrey Botanical Club 30: 544-563.

Fulford, M.H. 1963. Manual of the leafy Hepaticae of Latin America - Part I. Memoirs of The New York Botanical Garden 11: $1-172$.

Fulford, M.H. 1966. Manual of the leafy Hepaticae of Latin America - Part II. Memoirs of The New York Botanical Garden 11: 173-276.

Fulford, M.H. 1968. Manual of the leafy Hepaticae of Latin America - Part III. Memoirs of The New York Botanical Garden 11: 277-392.

Fulford, M.H. 1976. Manual of the leafy Hepaticae of Latin America - Part IV. Memoirs of The New York Botanical Garden 11: 393-535.

Ganacevich, N.A. \& Mello, Z.R. 2006. Briófitas da Biquinha de Anchieta, São Vicente, São Paulo, Brasil. Boletim do Instituto de Botânica 18: $75-84$

Germano, S.R. \& Pôrto, K.C. 2005. A Bryophyte Checklist of the Ecological Reserve of Gurjaú, Pernambuco, Brazil. Tropical Bryology 26: 1-12.

Gradstein, S.R. \& Costa, D.P. 2003. The Hepaticae and Anthocerotae of Brazil. Memoirs of the New York Botanical Garden 87: 1-318.

Gradstein, S.R.; Churchill, S.P. \& Salazar Allen, N. 2001. Guide to the Bryophytes of Tropical America. Memoirs of the New York Botanical Garden 86: 1-577.

Joyce, M.V.; Mello, Z R. \& Yano, O. 2006. Briófitas da Ilha de Palmas, Guarujá, São Paulo, Brasil. Boletim do Instituto de Botânica 18: 101-109.

Kron, K.A. 1988. Taxonomic study of Venezuelan members of Frullania Raddi subg. Meteoriopsis Spruce. The Journal of the Hattori Botanical Laboratory 64: 347-358.

Molinaro, L.C. \& Costa, D.P. 2001. Briófitas do arboreto do Jardim Botânico do Rio de Janeiro. Revista do Jardim Botânico do Rio de Janeiro 52: 107-124.

Moura, F.B.P. 2006. A Mata Atlântica em Alagoas. Maceió, Editora da UFAL.

Oliveira, J.R.M.; Alvarenga, L.D.P. \& Pôrto, K.C. 2006. Briófitas da Estação Ecológica de Águas Emendadas, Distrito Federal, material coletado por Daniel Moreira Vital. Boletim do Instituto de Botânica 18: 181-195.

Paixão, R.C. \& Mello, Z.P. 2006. Brioflora ao longo da Trilha do Costão, Peruíbe, São Paulo, Brasil. Boletim do Instituto de Botânica 18: 85-90.

Patrus, P. \& Starling, M.F.V. 2006. Briófitas da Serra do Curral, Belo Horizonte, Minas Gerais, Brasil. Boletim do Instituto de Botânica 18: 123-129.

Pôrto, K.C. \& Germano, S.R. 2002. Biodiversidade e importância das briófitas na conservação dos ecossistemas naturais de Pernambuco. Pp. 125-152. In: M. Tabarelli \& J.M.C. Silva (eds.). Diagnóstico da Biodiversidade de Pernambuco. SECTMA - Secretaria de Ciência, Tecnologia e Meio Ambiente, Recife.

Pôrto, K.C., Alvarenga, L.D.P. \& Santos, G.H.F. 2006. Briófitas. Pp. 123-146. In: K.C. Pôrto, M. Tabarelli \& J.S. Almeida-Cortez (orgs.). Diversidade Biológica e Conservação da Floresta Atlântica ao Norte do Rio São Francisco. Brasília, Ministério do Meio Ambiente/SBF.

Reiner-Drehwald, M.E. \& Goda, A. 2000. Revision of the genus Crossotelejeunea (Lejeuneaceae, Hepaticae). The Journal of the Hattori Botanical Laboratory 89: 1-54.

Schuster, R.M. 1992. The Hepaticae and Anthocerotae of North America. Chicago, Field Museum of Natural History, v. 5.

Stotler, R.E. 1970. The Genus Frullania in Latin America. Nova Hedwigia 18: 397-555.

Tixier, P. 1991. Bryophyta exótica - 9. Quelques Lejeuneaceae (Hepatiques) nouvelles pour l'Amérique du Sud. Candollea 46: 267-302. 
Valente, E.B. \& Pôrto, K.C. 2006a. Briófitas do afloramento rochoso na Serra da Jiboia, municipio de Santa Teresinha, Bahia, Brasil. Boletim do Instituto de Botânica 18: 207-211.

Valente, E.B. \& Pôrto, K.C. 2006b. Novas ocorrências de hepáticas (Marchantiophyta) para o Estado da Bahia, Brasil. Acta Botanica Brasilica 20: 195-201.

Veloso, H.P.; Rangel Filho, A.L.R. \& Lima, J.C.A. 1991. Classificação da vegetação brasileira adaptada a um sistema universal. Rio de Janeiro, Instituto Brasileiro de Geografia e Estatística.

Vilas Bôas-Bastos, S.B.; Bastos, C.J.P. \& Ballejos, J. 2006. Novas ocorrências de hepáticas (Marchantiophyta) para o Estado da Bahia, Brasil. Boletim do Instituto de Botanica 18: 142-147.

Yano, O. 1981. A checklist of Brazilian mosses. The Journal of the Hattori Botanical Laboratory 50: 279-456.

Yano, O. 1984. Checklist of Brazilian liverworts and hornworts. The Journal of the Hattori Botanical Laboratory 56: 481-548.

Yano, O. 1989. An additional checklist of Brazilian bryophytes. The Journal of the Hattori Botanical Laboratory 66: 371-434.

Yano, O. 1995. A new additional annotated checklist of brazilian bryophytes. The Journal of the Hattori Botanical Laboratory 78: $137-182$.
Yano, O. 2004. Novas ocorrências de briófitas para vários estados do Brasil. Acta Amazonica 34: 559-576.

Yano, O. 2005. Adição às Briófitas da Reserva Natural da Vale do Rio Doce, Linhares, Espírito Santo, Brasil. Boletim do Museu de Biologia Mello Leitão, Nova Série, 18: 5-48.

Yano, O. 2006. Novas adições as briófitas brasileiras. Boletim do Instituto de Botanica 18: 229-233.

Yano, O. \& Costa, D.P. 2000. Flora dos Estados de Goiás e Tocantins: Criptógamos: Briófitas. v.5. Goiânia, Editora da UFG.

Yano, O. \& Câmara, P.E. 2004. Briófitas de Manaus, Amazonas, Brasil. Acta Amazonica 34: 445-457.

Yano, O. \& Bordin, J. 2006. Novas ocorrências de briófitas para o Rio Grande do Sul, Brasil. Boletim do Instituto de Botânica 18: 111-122.

Yano, O. \& Peralta, D.F. 2005. Hepáticas (Marchantiophyta) de Mato Grosso, Brasil. Hoehnea 32: 185-205.

Yano, O. \& Peralta, D.F. 2006. Briófitas coletadas por Daniel Moreira Vital no Estado da Bahia, Brasil. Boletim do Instituto de Botânica 18: 33-73.

Yano, O. \& Peralta, D.F. 2007. Novas ocorrências de briófitas para os Estados de Alagoas e Sergipe, Brasil. Arquivos do Museu Nacional 64: 287-297. 